\title{
Influence of Concomitant Injuries on Outcome of Traumatic Brain Injury
}

\author{
Md Aminul Islam ${ }^{1 *}$, Shamantha Afreen², Al Amin Salek²
}

\begin{abstract}
:
Traumatic brain injury (TBI) is a leading cause of death and disability in trauma patients. Patients with TBI frequently sustain concomitant injuries in extra cranial regions. The effect of severe extracranial injury (SEI) on the outcome of TBI is controversial. We retrospectively enrolled 129 patients with head injury Total patients were dichotomized into isolated TBI $(n=90)$ and TBI associated with SEI $(n=39)$. The differences in severity and outcome between these two groups were analyzed. Mortality was $8.89 \%$ in the isolated TBI group and $20.28 \%$ in TBI with SEI group $(P=0.0719)$, but the Glasgow Outcome Scale (GOS) in terms of Good Recovery (GR) was more in the isolated TBI group (P= 0.0004). Adjusting for age, GCS, and length of hospital stay, SEI was a strong prognostic factor for mortality. It is important to manage not only the brain but the whole body in the treatment of TBI patients with SEI.
\end{abstract}

Key words: traumatic brain injury, extracranial injury, mortality, prognosis.

\section{Introduction:}

Traumatic brain injury (TBI) is the leading cause of trauma death and long-term disability among adult population below 40 years old ${ }^{1}$. Primary and secondary brain injuries such as decreased cerebral blood flow, hypotension, increased intracranial pressure and presence of medical complications are all known to affect medical and functional outcomes ${ }^{2-4}$. Apart from the primary and secondary brain injuries, concomitant injuries suffered during TBI are also known to have an aggravating effect on the patients' outcome. This is especially evident in the mortality outcome which is commonly associated with more severe concomitant injuries .$^{1,5,6}$ Overall, $78 \%$ of all patients with TBI suffer additional injuries. ${ }^{7,8}$ such as limb fractures, cardiopulmonary or visceral injuries, spinal cord injuries, peripheral nerve injuries and limb amputations 9 . Many authors have reported that Severe Extracranial Injury (SEI) with concomitant head injury is associated with high mortality rates. ${ }^{10-12}$ The influence of extracranial injury on the outcome of TBI remains controversial. ${ }^{13-16}$ In patients with brain injury, concomitant injuries in extracranial sites cause decreased cerebral blood flow and/or coagulopathy due to massive hemorrhage as secondary brain damage. ${ }^{17} \mathrm{We}$ conducted a retrospective observational single-center study of TBI with concomitant injuries.

1 Head of Neurosurgery center, Combined Military Hospital (CMH), Dhaka.

2. Consultant Neurosurgeon, Dept. of Neurosurgery, CMH, Dhaka.

\section{* Corresponding Author:}

Prof Col Md Aminul Islam Head of Neurosurgery center $\mathrm{CMH}$, Dhaka

E-mail aminulns@yahoo.com Cell: 01710840033

\section{Materials and Method:}

This study was conducted in the Emergency \& Casualty dept., $\mathrm{CMH}$, Dhaka, a tertiary referral center for armed forces during the year 2018. The hospital has a well-equipped Emergency Department (ED) with readily available surgical staff members and facilities such as X-rays, ultrasounds and computed tomography (CT). All patients presented to the ED with suspected diagnosis of TBI were reviewed by the neurosurgical team.

In this study, the initial selection of patients was done retrospectively by going through the hospital records of all patients presented to the ED, for first episode of TBI between Jan 2019 and Dec 2019. Patients $<18$ yrs age, who were dead on arrival at the ED or had preexisting cognitive, behavioral or physical disabilities before the presenting TBI were excluded from the study. After the initial exclusion, the remaining hospital charts were further reviewed for the patients' demographic profile, Glasgow Coma Scale (GCS) measurement on arrival at the ED, mechanism of TBI, early CT brain scan findings, presence of hypoxia, hypotension and concomitant injuries; and the therapeutic neurosurgical intervention performed (e.g. craniotomy and clot evacuation).GCS scores were used to divide patients into mild (13-15), moderate (9-12) or severe (3-8) TBI. CT brain scan findings were documented based on the radiologist reports provided in the hospital charts and divided into two groups. A normal CT brain scan finding was considered as showing no visible acute TBI changes whereas an abnormal CT brain scan finding was considered as showing some visible changes due to the acute TBI. Concomitant injuries are injuries affecting other body regions presented at the same time with the TBI. Each TBI and concomitant injury was rated according to Abbreviated Injury Scale (AIS) criteria (1998 updated version of AIS).$^{18}$ AIS is an anatomical scoring system which grades individual injuries and forms the basis of Injury Severity Score. The scale ranges from 1 to $6,1=$ minor, $2=$ moderate, $3=$ serious, $4=$ severe, $5=$ critical and $6=$ fatal injuries. Only AIS score for body regions of each individual injury was 
documented. In this study, patients with concomitant injuries of AIS grade3 were grouped together as having clinically significant concomitant injuries for the purpose of comparison on the long-term functional outcome. WE defined severe extra cranial injury (SEI) as AIS $\geq 3$ injuries in the face, chest, abdomen, and pelvis/extremities ${ }^{19}$

A functional assessment was conducted by two designated clinicians in the outpatient clinic using Glasgow Outcome Scale (GOS) as the outcome measure. GOS classifies functional level into five categories: death, vegetative state, severe disability, moderate disability and total recovery ${ }^{20}$.

A $p$ value of less than 0.05 was considered statistically significant. All statistical analysis was performed using SPSS version 17.

\section{Results}

Total 129 patients with head injury (HI) were studied retrospectively after fulfilling inclusion criteria. The TBI with SEI group comprised 39 patients accounting for $30.23 \%$ of the total. The reaming 90 patients belonged to Isolated Head Injury group. The mean age was in the $18-40$ yrs. in both groups, and though a male predominance was seen, no significant difference was found in this between them (Table 1). Regarding the mechanism of injury, in the isolated TBI group traffic accidents predominated (80\%), with fall implicated in many of the remaining cases. The diagnosis of head injuries was classified as epidural /subdural hematoma, brain contusion, traumatic subarachnoid hemorrhage, and diffuse axonal injury.

The frequency of acute hematoma in the isolated TBI group was significantly higher than that in TBI with SEI group (Table 1). In the TBI with SEI group, in addition to the head, the chest, abdomen, extremities, and pelvis often showed injuries, and so the frequency of surgery in this group was high. The incidence of epidural and/or subdural hematoma was significantly higher in the isolated TBI group, whereas DAI was significantly higher in the TBI with SEI group.

Table 1: Baseline characteristics of 129 study patients

\begin{tabular}{lccc}
\hline & $\begin{array}{c}\text { Total } \\
\mathrm{n}=129\end{array}$ & $\begin{array}{c}\text { Isolated TBI } \\
\mathrm{n}=90\end{array}$ & $\begin{array}{c}\text { TBI with SEI } \\
\mathrm{n}=39\end{array}$ \\
\hline Age & & 32 & 23 \\
$18-40$ & 55 & 35 & 15 \\
$41-60$ & 40 & 21 & 13 \\
$>60$ & 34 & & \\
Gender & 114 & 65 & 49 \\
Male & 15 & 9 & 6 \\
Female & 103 & 57 & 46 \\
Mechanism & 11 & 4 & 7 \\
RTA & & & \\
Fall & & 57 & \\
\hline
\end{tabular}

\begin{tabular}{lccc}
\hline Physical assault & 8 & 3 & 5 \\
others & 7 & 2 & 5 \\
Type of HI & & 48 & 17 \\
ASDH\&/ EDH & 65 & 7 & 8 \\
tSAH & 15 & 8 & 17 \\
Contusion & 25 & 4 & 15 \\
DAI & 19 & 1 & 4 \\
Others & 5 &
\end{tabular}

RTA: Road traffic accident, ASDH: acute subdural hematoma, EDH: epidural hematoma, DAI: diffuse axonal injury, SEI: severe extracranial injuries, tSAH: traumatic subarachnoid hemorrhage, TBI: traumatic brain injury.

The frequency and distribution of extracranial concomitant injuries according to the AIS score are illustrated in Table 2. Among the AIS grade 3, injuries in extremities were more common.

Table 2. Frequency of extracranial concomitant injuries according to AIS in all the patients

\begin{tabular}{lcccc}
\hline & Face & Chest & Abdomen & extremities \\
\hline AIS1 & 4 & 3 & 1 & 0 \\
AIS2 & 7 & 4 & 2 & 3 \\
AIS3 & 3 & 5 & 2 & 15 \\
AIS4 & 1 & 1 & 1 & 3 \\
\hline
\end{tabular}

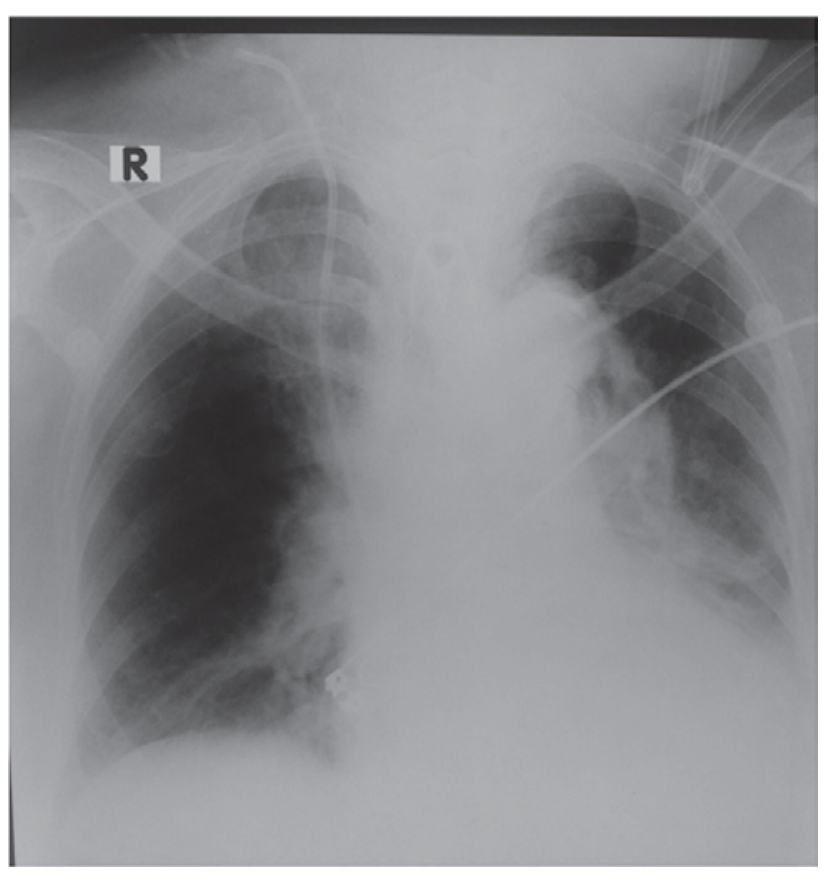

Figure: A 
Bangladesh Crit Care J March 2020; 8 (1): 24-28

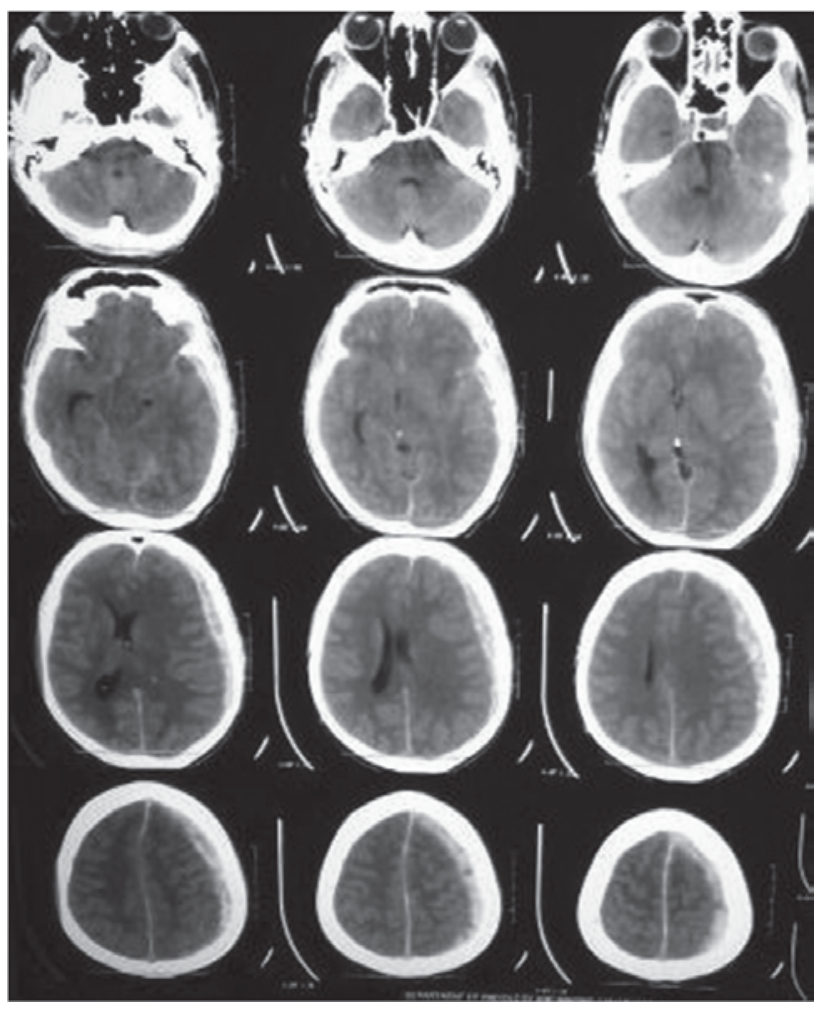

Figure: B

Figure A showing concomitant chest injury associated with head injury

Figure B showing left sided ASDH with mid line shifting

Table 3 shows mortality to be $8.89 \%$ in the isolated TBI group, and $20.28 . \%$ in the TBI with SEI group $(\mathrm{P}=.0719)$. On the other hand, regarding GOS, in the isolated TBI group, GR was seen in 49 (54.44\%) while in the TBI with SEI group GR was $20.51 \%$. When the functional outcome including death was compared using GOS it was found to be better in the isolated TBI group than in the TBI with SEI group $(\mathrm{P}=.0004)$

Table 3: Outcome:

\begin{tabular}{lccc}
\hline GOS & $\begin{array}{c}\text { Isolated TBI } \\
\mathrm{n}=90\end{array}$ & $\begin{array}{c}\text { TBI with SEI } \\
\mathrm{n}=39\end{array}$ & $\mathrm{P}$ \\
\hline GR & $49(54.44 \%)$ & $8(20.51 \%)$ & .0004 \\
MD & $17(18.8 \%)$ & $7(17.94 \%)$ & .9085 \\
SD & $15(16.66 \%)$ & $10(25.64 \%)$ & .2478 \\
PVS & $1(1.11 \%)$ & $3(7.69 \%)$ & .0485 \\
D & $8(8.89 \%)$ & $11(28.20 \%)$ & .0719 \\
\hline
\end{tabular}

D: dead, GCS: Glasgow Coma Scale, GR: good recovery, MD: moderate disability, PVS: persistent vegetative state, SD: severe disability, SEI: severe extracranial injury, TBI: traumatic brain injury.

\section{Discussion}

We found that younger men have higher rates of severe TBI due to RTAs. This can be due to pattern of their lifestyles, work, and activities; eg, their high risk job and more use of motor vehicle ${ }^{21}$ This result is in accordance with several previous studies. ${ }^{22-25}$ However in a number of studies a greater incidence of HI was observed in elderly patients. ${ }^{26}$ RTAs are the most common cause of $\mathrm{TBI}^{27,28}$ which is in accordance with our study .This may be due to the fact that many people are not following traffic rules. Despite the fact that wearing helmet is mandatory, a number of cases found not following the rules. Although coexistence of TBI and concomitant injuries is common, its influence on the long-term functional outcome has not been widely studied. Previous reports focused on the occurrence of specific concomitant injuries with TBI, for example extremity injuries, spinal cord injuries or cranial injuries ${ }^{29-32}$.

Lower extremity injuries were common in this study, second only to facial injuries. The high frequency of lower extremity concomitant injuries is not surprising since $79.84 \%$ of the TBI etiology in our study population was related to motor vehicle collision. The high-impact trauma associated with motor vehicle collision made the extremities vulnerable to fracture, crush or amputation injury. This is consistent with a high incidence of concurrent lower extremity injuries noted in multiple trauma patients with TBI ${ }^{32}$. Chest, abdomen and spine injuries were less common in this study, but they were among the more severe concomitant injuries reported. Chest injuries were the most common extracranial concomitant injuries measuring AIS grade 3. The presence of hemothorax or pneumothorax are among the factors classifying chest injuries measured at AIS grade three. ${ }^{33}$

Extracranial concomitant injuries were found to have significant influence on functional outcome among TBI patients. The study demonstrated that although the initial TBI is mild, clinically significant concomitant injuries influenced the disability outcome in the long term.

Extracranial concomitant injuries of AIS grade3 remained significant predictors for unfavorable outcome. The main reasons for an increased risk of developing long term disabilities in the presence of severe extracranial concomitant injuries are due to physical limitation and mobility restriction. Severe extremity injuries, which can affect physical function and mobility, were more common than severe spinal or abdominal injuries in this study. Previous studies have revealed that severe injuries of the lower extremities can affect long-term functional outcome regardless of treatment options. ${ }^{33,34}$

Old age above 40 is another significant predictor for disability in this study as previously demonstrated in other studies ${ }^{35-37}$. Older individuals above the age of 40 were found to have worse long-term recovery and they were more likely to have functional decline ${ }^{37}$.

Our study has several limitations. First, the number of patients was relatively small which reduced the overall statistical strength of the study. A larger number may show significant association of severe AIS among the more severe TBI patients. Second, the selection of patients was done retrospectively. Other factors which can contribute to 
functional outcome such as pre-injury education and premorbid medical comorbidities were also not analyzed. Future prospective studies with a larger sample size should be conducted to ascertain a better understanding on the influence of extracranial concomitant injuries to the long-term functional outcome in patients with different TBI severities.

\section{Conclusion}

SEI is an independent prognostic factor for mortality in TBI patients. Presence of extracranial concomitant injuries play an important role in prognosis. Among those who survived the initial TBI, the presence of extracranial concomitant injuries influenced the long-term functional outcome resulting in moderate and severe disabilities. This finding suggests that disability outcome depends on a more complex interaction between the severity of concomitant injuries and TBI itself. Therefore, careful assessment is necessary for early diagnosis of concomitant injuries in patients with head injury.

\section{References}

1. Lefering R, Bouillon LR, Neugebauer EA. Head injury and outcome: what influence do concomitant injuries have? J Trauma 2008; 65:1036-43.

2. Ojha BK, Jha DK, Kale SS, Mehta VS. Trans-cranial doppler in severe head injury: evaluation of pattern of changes in cerebral blood flow velocity and its impact on outcome. SurgNeurol 2005; 64:174-9.

3. Manley G, Knudson M, Morabito D, et al. Hypotension, hypoxia and head injury. Arch Surg 2001; 136:1118-23.

4. Cormio M, Robertson CS, Narayan RK. Secondary insults to the injured brain. J ClinNeurosc 1997; 4:132-48.

5. MRC CRASH Trial Collaborators, Perel PA, Arango M, et al. Predicting outcome after traumatic brain injury: practical prognostic models based on large cohort of international patients. BMJ 2008; 336:425-9.

6. Sarrafzadeh AS, Peltonen EE, Kaisers U, et al. Secondary insults in severe head injury - do multiply injured patients do worse? Crit Care Med 2001; 29:1116-23.

7. Rickels E, Wild K, Wenzlaff P. Head injury in Germany: a population-based prospective study on epidemiology, causes, treatment and outcome of all degrees of head-injury severity in two distinct areas. Brain Inj. 2010; 24(12):1491-504.

8. Leong BK, Mazlan M, Abd Rahim RB, Ganesan D. Concomitant injuries and its influence on functional outcome after traumatic brain injury. DisabilRehabil. 2013; 35(18):1546-51.

9. Chua KS, Ng YS, Yap SG, Bok CW. A brief review of traumatic brain injury rehabilitation. Ann Acad Med Singapore 2007; 36:31-42.

10. McMahon CG, Yates DW, Campbell FM, Hollis S, Woodford M: Unexpected contribution of moderate traumatic brain injury to death after major trauma. J Trauma 47: 891-895, 1999

11. Gennarelli TA, Champion HR, Copes WS, and Sacco WJ: Comparison of mortality, morbidity, and severity of 59,713 head injured patients with 114,447 patients with extracranial injuries. J Trauma 37: 962-968, 1994

12. Patel HC, Bouamra O, Woodford M, King AT, Yates DW, Lecky FE; Trauma Audit and Research Network: Trends in head injury outcome from 1989 to 2003 and the effect of neurosurgical care: an observational study. Lancet 366: 1538-1544, 2005
13. Jacobs B, Beems T, Stulemeijer M, et al.: Outcome prediction in mild traumatic brain injury: age and clinical variables are stronger predictors than CT abnormalities. J Neurotrauma 27: 655-668, 2010

14. Lefering R, Paffrath T, Linker R, Bouillon B, Neugebauer EA; Deutsche GesellschaftfürUnfallchirurgie/ German Society for Trauma Surgery: Head injury and outcome-what influence do concomitant injuries have? J Trauma 65: 1036-1044, 2008

15. Ho KM, Burrell M, Rao S: Extracranial injuries are important in determining mortality of neurotrauma. Crit Care Med 38: $1562-1568,2010$

16. Sarrafzadeh AS, Peltonen EE, Kaisers U, Küchler I, Lanksch WR, and Unterberg AW: Secondary insults in severe head injury-do multiply injured patients do worse? Crit Care Med 29: 1116-1123, 2001

17. McDonald SJ, Sun M, Agoston DV, Shultz SR: The effect of concomitant peripheral injury on traumatic brain injury pathobiology and outcome. J Neuroinflammation

18. Association for the advancement of automotive medicine annual meeting. Accident Analysis \& Prevention. 1990; 22(2):191-192.

19. Watanabe $\mathrm{T}$, Kawai Y, Iwamura A, Maegawa N, Fukushima H, Okuchi K, and Outcomes after Traumatic Brain Injury with Concomitant Severe Extracranial Injuries. Neurol Med Chir (Tokyo). 2018 Sep; 58(9): 393-399. doi: 10.2176/nmc.oa.2018-0116

20. Jennett B, Bond M. Assessment of outcome after severe brain damage. Lancet $1975 ; 1: 480-4$.

21. Roozenbeek B, Maas AI, Menon DK. changing patterns in the epidemiology of traumatic brain injury. Nat Rev Neurol2013; 9:231-6.

22. Atci IB, Yilmaz H, Kara D, Yilmaz N, Bitlisli H, Ayden O, et al. Retrospective analysis of 3524 head trauma $\quad \mathrm{p}$ a $\mathrm{t}$ i e $\mathrm{n} \mathrm{t}$ s admitted to the emergency department as epidemiological approach to head traumas in our region. Acta Med Mediterranea2015; 31:825-8

23. Grigorakos L, Alexopoulou A, Tzortzopoulou K, Stratouli S , Chroni D, PapadakiE, et al. Predictors of outcome in patients with severe traumatic brain injury. J NeurosciClinRes $2016 ; 1: 1$.

24. Saini NS, Rampal V, Dewan Y, Grewal SS. Factors predicting outcome in patients with severe head injury: Multivariate analysis. Indian J Neurotrauma $\quad 2012 ; 9: 45-8$.

25. .BaumJ,Entezami P, Shah K, Medhkour A. Predictors of outcomes in traumatic brain injury. World Neurosurg2016:90 525-9

26. Roozenbeek B, Maas AI, Menon DK. Changing patterns in the epidemiology of traumatic brain injury. Nat Rev Neurol2013;9:231-6.

27. Brazinova A, Rehorcikova V, Taylor MS, Buckova V, Majdan M, PsotaM,et al. Epidemiologyof traumatic brain injury in Europe: A living systematic review. J Neurotrauma2016.

28. Peeters W, van den Brande R, Polinder S, Brazinova A, Steyerberg $\mathrm{E}$, Lingsma $\mathrm{H}$, et al. Epidemiology of traumatic brain injury in Europe. ActaNeurochir2015; 157:1683-96.

29. Macciocchi SN, Bowman B, Coker J, et al. Effect of co-morbid traumatic brain injury on functional outcome of persons with spinal cord injuries. Am J Phys Med Rehabil 2004; 83:22-6.

30. Macciocchi S, Seel RT, Thompson N, et al. Spinal cord injury and co-occurring traumatic brain injury: assessment and incidence. Arch Phys Med Rehabil 2008; 89:1350-7.

31. Pappachan B, Alexander M. Correlating facial fractures and cranial injuries. J Oral MaxillofacSurg 2006; 64:1023-9. 
Bangladesh Crit Care J March 2020; 8 (1): 24-28

32. Grotz MR, Giannoudis PV, Pape HC, et al. Traumatic brain injury and stabilisation of long bone fractures: an update. Injury 2004; 35:1077-86.

33. Holtslag H, Buskens E, Rommers C, et al. Long-term outcome after lower extremity injuries in severely injured patients. Eur J Trauma 2006; 32:365-73.

34. MacKenzie EJ, Bosse MJ, Pollak AN, et al. Long-term persistence of disability following severe lower-limb trauma: results of a sevenyear follow-up. J Bone Joint Surg Am 2005; 87:1801-9.
35. Susman M, DiRusso SM, Sullivan T, et al. Traumatic brain injury in the elderly: increased mortality and worse functional outcome at discharge despite lower injury severity. J Trauma 2002; 53:219-24.

36. Marquez de la Plata CD, Hart T, Hammond FM, et al. Impact of age on long-term recovery from traumatic brain injury. Arch Phys Med Rehabil 2008; 89:896-903.

37. Mendelow AD. The effect of age on outcome following severe head injury for patients in coma from the outset: a mitochondrial DNA degradation phenomenon? Ind J Neurotrauma 2006; 3:95-8. 